Pacific Journal of Mathematic 


\title{
MATRIX CORRESPONDENCES OF PLANE PARTITIONS
}

\author{
EMDEN R. GANSNER
}

\begin{abstract}
Three correspondences between nonnegative integer arrays and plane partitions, due to Burge, Knuth, and Hillman and Grassl, are investigated. A variety of parallel and complementary properties are derived for the first two. In particular, the images of an array under the correspondences are characterized in terms of certain sets of "paths" in the arrays. The correspondences are also related to each other through the action of the dihedral group on rectangular arrays. Finally, the Hillman-Grassl correspondence is similarly characterized in terms of sets of paths and is shown to be an extension of Burge's correspondence.
\end{abstract}

One of the most elegant developments in the study of plane partitions has been the invention of constructive correspondences between nonnegative integer arrays and certain forms of plane partitions. Besides providing potent tools for investigating these partitions, they also serve to connect the theory of plane partitions with those of symmetric functions and group representations (cf. [23]), among others.

Here we limit our attention to three correspondences, four if we include the correspondence of Schensted [20] between permutations and pairs of standard Young tableaux. This bijection was extended by Knuth [15] to a correspondence $K$ between matrices and pairs of generalized Young tableaux or, as we shall call them, column-strict plane partitions. In turn, Burge [4] defined a new correspondence $B$ based on a variation of the original construction that was also investigated by Schensted and Knuth. The final correspondence to be considered, due to Hillman and Grassl [13], is entirely different in definition, yet, as will be shown, is very much related to $B$ and $K$.

Indeed, a presentation of these correspondences in a fairly unified setting is the main purpose of this paper, in which their interconnections and parallel developments are emphasized. The first three sections are devoted to preliminaries and the definitions of $B$ and $K$. Schensted's correspondence is introduced in $\S 4$ as a restriction of $K$. However, it is also noted that both $B$ and $K$ factor naturally through Schensted's map, implying that the properties of the latter correspondence will occur in some form as properties of $B$ and $K$. This fact has been common knowledge for some time; here we present an explicit factorization.

Greene [11] has given a means for determining the shape of the 
image Young tableaux in Schensted's correspondence. We extend his results to $B$ and $K$, and then use them to give a complete characterization of the plane partitions $B(M)$ and $K(M)$ in terms of sets of paths in the matrix $M$ (Theorems 5.4 and 5.5). These results can be quite useful in the enumeration of plane partitions [10] and make obvious the symmetry and other properties of the correspondences. For example, among several properties of $B$ and $K$ given in $\S 6$, we note that $B$, when restricted in a natural way to acting on a sequence of integers, is equal to $K$ acting on the sequence in the opposite order. This well-known fact follows trivially from Theorem 5.4.

In $\S \S 7$ and 8 we introduce two operations due to Schützenberger [21] which enable us to compute the effect under $B$ and $K$ of the dihedral group, generated by rotations and transpositions, acting on a matrix. The results obtained present another interlacing of the correspondences.

The Hillman-Grassl correspondence between nonnegative integer arrays and reverse plane partitions is considered in the final two sections. We note that this correspondence can be characterized in terms of certain sets of paths in integer arrays in the same manner as $B$, thereby implying that this correspondence can be considered an extension of the correspondence of Burge.

The author wishes to express his thanks to Richard P. Stanley, whose information, suggestions and warm encouragement did so much to make this paper possible.

1. Shapes and plane partitions. A shape $\lambda$ is a finite nonincreasing sequence $\lambda_{1} \geqq \lambda_{2} \geqq \cdots \geqq \lambda_{p}$ of positive integers. The $\lambda_{i}$ are called the parts of $\lambda$. We let $\rho(\lambda)=p$, the number of parts in $\lambda$, and we define the norm of $\lambda$ to be $\sigma(\lambda)=\lambda_{1}+\cdots+\lambda_{p}$. The graph $\Gamma(\lambda)$ of $\lambda$ is the set of all $(i, j)$ with $1 \leqq i \leqq \rho(\lambda), 1 \leqq j \leqq \lambda_{i}$.

If $\lambda$ is a shape, let $\Gamma^{\prime}$ be the set of all $(i, j)$ such that $(j, i)$ is in $\Gamma(\lambda)$. If we let $\lambda^{\prime}$ be the unique shape such that $\Gamma\left(\lambda^{\prime}\right)=\Gamma^{\prime}$, we call $\lambda^{\prime}$ the conjugate of $\lambda$. If $\lambda=\lambda^{\prime}$, we say that $\lambda$ is symmetric. If $\lambda$ is a constant sequence, $\lambda_{1}=\lambda_{2}=\cdots=\lambda_{p}=n, \lambda$ is said to be rectangular, and we can denote it by $\left(n^{p}\right)$, signifying $p$ parts equal to $n$.

Obviously, shapes are nothing more than partitions of integers, which have their own extensive theory (cf. [1]). For our purposes, however, we view them as a base from which to construct plane partitions, extending from one-dimensional arrays to two-dimensional ones. From another viewpoint, given a shape, we can view its graph as an order ideal (o-ideal, $M$-closed subset) of $\boldsymbol{P}^{2}$, the direct product of the chain of natural numbers $\boldsymbol{P}$ with itself. (See [3] or [5] for the relevant definitions.) This leads to the consideration 
of order ideals in $\boldsymbol{P}^{3}$ and plane partitions.

Let $\lambda$ be a shape. A plane partition $P=\left(p_{i j}\right)$ of shape $\lambda$ is an array of positive integers indexed by $\Gamma(\lambda)$ such that

$$
p_{i j} \geqq p_{i j+1} \quad \text { and } \quad p_{i j} \geqq p_{i+1 j},
$$

whenever both sides are defined. As with shapes, the $p_{i j}$ are the parts of $P$ and the norm of $P, \sigma(P)$, is the sum of all the parts. If $k$ is an integer, $1-\rho(\lambda) \leqq k \leqq \lambda_{1}-1$, the $k$-diagonal of $P$ is the nonincreasing sequence induced by the $p_{i j}$ with $j-i=k$. We define $T P=\left(q_{i j}\right)$ to be the plane partition of shape $\lambda^{\prime}$ where $q_{i j}=p_{j i}$. If $T P=P$, we say that $P$ is symmetric.

We can extend some of these definitions in an obvious manner to more general arrays. If $M=\left(m_{i j}\right)$ is an $r \times c$ matrix, let $\sigma(M)=$ $\sum m_{i j}$, summed over all $i$ and $j$, and let $T M$ be the transpose of $M$.

Certain special types of plane partitions will play an important role in the sequel. If the parts of a plane partition $P=\left(p_{i j}\right)$ strictly decrease down the columns, i.e., $p_{i j}>p_{i+1 j}$, then $P$ is a column-strict plane partition. If $P$ has shape $\lambda$ and its parts consist of all the integers from 1 to $\sigma(\lambda)$, we call $P$ a standard Young tableau.

Having defined plane partitions, we can consider reversing the inequalities. A reverse plane partition of shape $\lambda$ is an array $P=\left(p_{i j}\right)$ of nonnegative integers indexed by $\Gamma(\lambda)$ such that

$$
p_{i j} \leqq p_{i+1 j} \text { and } \quad p_{i j} \leqq p_{i j+1} .
$$

All the other definitions given concerning plane partitions are precisely the same for reverse plane partitions.

2. The Frobenius construction. Frobenius [6] devised a bijection between shapes and pairs of shapes whose parts strictly decrease. Bender and Knuth [2] extended this construction to give a bijection between plane partitions and pairs of column-strict plane partitions of the same shape. We will have a great deal of use for a variation of their construction.

Let $P=\left(p_{i j}\right)$ and $Q=\left(q_{i j}\right)$ be two column-strict plane partitions of the same shape $\lambda$. We define an array $A=\left(a_{i j}\right)$ as follows. Let $\left(a_{i i}, a_{i+1}, a_{i+2}, \cdots\right)$ be the conjugate of row $i$ of $Q$, viewed as a shape. Let $\left(a_{i i}, a_{i+1 i}, a_{i+2}, \cdots\right)$ be the conjugate of row $i$ of $P$. Since $a_{i i}=\lambda_{i}$, the construction is well-defined.

It is easy to verify that $A$ is a plane partition, and that, given any plane partition, this process can be reversed. Thus, we have a bijection, which we call the Frobenius construction, between plane partitions $A=\left(a_{i j}\right)$ and pairs of column-strict plane partitions $P=$ $\left(p_{i j}\right)$ and $Q=\left(q_{i j}\right)$ of the shape $\lambda$. The bijection is characterized by 


$$
a_{i j}= \begin{cases}\mid\left\{k: p_{j k} \geqq i-j+1\right\} & \text { for } j \leqq i \\ \left|\left\{k: q_{i k} \geqq j-i+1\right\}\right| & \text { for } i \leqq j\end{cases}
$$

where $|V|$ denotes the cardinality of the set $V$. This follows immediately from the definition of the conjugate of a shape.

In addition, the number of rows (columns) of $A$ equals the largest part in $P(Q)$; the 0 -diagonal of $A$ equals $\lambda$; and $A$ is symmetric if and only if $P$ equals $Q$.

As an example of this construction, the column-strict plane partitions

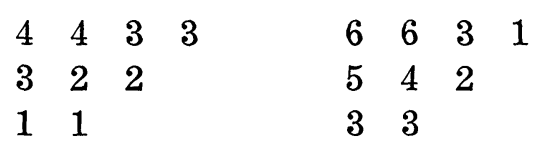

correspond to the single plane partition

$\begin{array}{llllll}4 & 3 & 3 & 2 & 2 & 2 \\ 4 & 3 & 3 & 2 & 2 & 1 \\ 4 & 3 & 2 & 2 & 2 & \\ 2 & 1 & & & & \end{array}$.

In the Bender-Knuth version of this construction, the plane partition derived from a pair of column-strict plane partitions is a "conjugate" of the plane partition derived using the construction given above. (For a detailed discussion of plane partition conjugates, see [18, pp. 176-181].) This conjugation disguises the geometric relations between the pair of plane partitions and the associated single plane partition. For example, symmetric plane partitions no longer correspond to a pair of equal column-strict plane partitions.

3. The correspondences of Burge and Knuth. Let $M=\left(m_{i j}\right)$ be an $r \times c$ matrix with nonnegative integer entries. Using $M$, we will see how to construct two pairs of column-strict plane partitions of the same shape, thereby defining the Burge and Knuth correspondences.

To the matrix $M$ we can correspond a two-line array

$$
\left(\begin{array}{llll}
a_{1} & a_{2} & \cdots & a_{N} \\
b_{1} & b_{2} & \cdots & b_{N}
\end{array}\right)
$$

in which $a_{1} \geqq a_{2} \geqq \cdots \geqq a_{N}, b_{i} \leqq b_{i+1}$ if $a_{i}=a_{i+1}$ and a column $\left(\begin{array}{l}a \\ b\end{array}\right)$ must appear in the array $m_{b a}$ times.

Using the two-line array, we inductively build two arrays $P$ and $Q$ of the same shape out of the $b_{i}$ 's and $a_{i}$ 's, respectively. We start by letting $P_{1}=\left(b_{1}\right)$ and $Q_{1}=\left(a_{1}\right)$. Now, assuming we have defined 
$P_{j}$ and $Q_{j}$, we insert $b_{j+1}$ into the first column of $P_{j}$ by placing it at the bottom of the column if $b_{j+1}$ is strictly smaller than every entry in the column. Otherwise, we use $b_{j+1}$ to replace the uppermost entry which is less than or equal to $b_{j+1}$. The entry replaced is inserted in the same manner into the second column. If any entry is replaced in that column, it is inserted into the third column. This process continues until an entry is placed at the bottom of a column or is placed in the first position of an empty column. This defines $P_{r+1}$. We obtain $Q_{r+1}$ by attaching $a_{r+1}$ to $Q_{r}$ in order that $P_{r+1}$ and $Q_{r^{+1}}$ have the same shape. Finally, if $N=\sigma(M)$, we let $P=P_{N}$ and $Q=Q_{N}$. Examples illustrating this process can be found in [4] and [10].

This construction is Burge's correspondence, originally presented in [4]. It is a variation of the "dual" correspondence of Knuth, which can be found in [15] along with the construction we call Knuth's correspondence. This can be defined in the same manner as Burge's correspondence, with the following modifications. As above, with the given matrix we correspond the two-line array in which $a_{1} \geqq a_{2} \geqq \cdots \geqq a_{N}$ and a column $\left(\begin{array}{l}a \\ b\end{array}\right)$ appears $m_{b a}$ times, but now, $b_{i} \geqq b_{i+1}$ whenever $a_{i}=a_{i+1}$. Second, instead of inserting entries down the columns, we now insert entries along the rows, either replacing the leftmost entry which is strictly less than the inserted entry or placing the inserted entry at the end of the row if every entry in the row is greater than or equal to it.

Concerning either correspondence, we immediately note that the number of $i$ 's in $P$ is $\sum_{j=1}^{c} m_{i j}$, the number of $j$ 's in $Q$ is $\sum_{i=1}^{r} m_{i j}$ and $\sigma(\lambda)=\sigma(M)$, where $\lambda$ is the shape of $P$ (or $Q)$. More importantly, we have the following fundamental result concerning these constructions.

THEOREM 3.1. Burge's and Knuth's correspondences are bijections between nonnegative integer matrices of size $r \times c$ and pairs of column-strict plane partitions $(P, Q)$ of the same shape such that the largest part in $P$ is at most $r$ and the largest part in $Q$ is at most $c$.

Proofs for the parts of this theorem can be found in [4], [7, pp. 43-56], [15], and [23], among other sources. We will also mention in the next section another approach to a proof of this theorem using a specialization of Knuth's correspondence.

If these correspondences are combined with the bijection given by the Frobenius construction, we obtain two bijections between matrices and plane partitions.

Theorem 3.2. The Burge and Knuth correspondences, when 
combined with the Frobenius construction, form bijections between the set of all $r \times c$ nonnegative integer matrices and the set of all plane partitions with at most $r$ rows and c columns.

We will be interested in these correspondences in their several forms. To simplify the notation, we let $B$ represent the Burge correspondence, regardless of whether the domain consists of matrices or two-line arrays and whether the range consists of pairs of columnstrict plane partitions or a single plane partition. When $B$ maps to a pair of column-strict plane partitions, we let $B^{1}$ be the projection of $B$ onto the first component of the pair and $B^{2}$ the projection onto the second. We allow $B^{1}$ to also operate on any finite sequence $\left(b_{1}, b_{2}, \cdots, b_{N}\right)$ of positive integers by defining

$$
B^{1}\left(b_{1}, b_{2}, \cdots, b_{N}\right)=B^{1}\left(\begin{array}{ccccc}
N & N-1 & \cdots & 2 & 1 \\
b_{1} & b_{2} & \cdots & b_{N-1} & b_{N}
\end{array}\right) .
$$

Finally, if $b$ is a positive integer and $P$ is a column-strict plane partition, we let $B_{b}(P)$ be the column-strict plane partition obtained from $P$ by inserting $b$ using the Burge insertion process. Similar conventions will be observed for the Knuth correspondence $K$.

4. The correspondence of Schensted. If $K$ is restricted to permutation matrices, we obtain the correspondence of Schensted between permutations and pairs of standard Young tableaux of the same shape. This bijection was explicitly defined by Schensted in [20], though it was essentially given by Robinson in $[19, \S 5]$. Using it, Knuth devised his correspondence, and from Knuth, Burge defined his.

To deal with Schensted's correspondence, we extend our notation one final step. Given a permutation $\pi$ on the set $\{1,2, \cdots, n\}$, we let

$$
K(\pi)=K\left(\begin{array}{cccc}
n & \cdots & 2 & 1 \\
\pi(n) & \cdots & \pi(2) & \pi(1)
\end{array}\right) .
$$

The same notation will also hold for $K^{1}$ and $K^{2}$.

All of the results that we shall give concerning $K$ have immediate corollaries pertaining to Schensted's correspondence. But, in a sense, the latter correspondence is not significantly less general than either $K$ or $B$. Both of these functions can be factored through the Schensted map, and their properties follow from its properties.

To see this, consider a two-line array used in the Burge correspondence:

$$
\left(\begin{array}{llll}
a_{1} & a_{2} & \cdots & a_{N} \\
b_{1} & b_{2} & \cdots & b_{N}
\end{array}\right)
$$


with $a_{i} \geqq a_{i+1}$ and $b_{\imath} \leqq b_{\imath+1}$ whenever $a_{i}=a_{i+1}$. From this array, we can obtain a permutation by replacing $\left(a_{1} a_{2} \cdots a_{N}\right)$ by $(N N-1 \cdots$ $21)$, and by replacing the leftmost minimum-sized entry in $\left(b_{1} b_{2} \cdots b_{N}\right)$ by 1 , the next leftmost minimum-sized entry by 2 , etc. For example, the array

$$
\left(\begin{array}{lllllllll}
3 & 3 & 3 & 3 & 2 & 2 & 1 & 1 & 1 \\
1 & 2 & 2 & 4 & 1 & 1 & 2 & 3 & 3
\end{array}\right)
$$

becomes the permutation

$$
\left(\begin{array}{lllllllll}
9 & 8 & 7 & 6 & 5 & 4 & 3 & 2 & 1 \\
1 & 4 & 5 & 9 & 2 & 3 & 6 & 7 & 8
\end{array}\right)
$$

We also need two functions to be able to obtain the original array from its derived permutation $\pi$. Define $\Phi_{a}(i)=a_{N-\imath+1}$ and $\Phi_{b}(i)=b_{N-j+1}$, where $i=\pi(j)$. Note that these two functions, besides being nondecreasing, satisfy the following properties:

$$
\begin{array}{lll}
\Phi_{a}(l+1)=\Phi_{a}(l) & \text { implies } & \pi(l+1)<\pi(l) ; \\
\Phi_{b}(l+1)=\Phi_{b}(l) & \text { implies } & \pi^{-1}(l+1)<\pi^{-1}(l) .
\end{array}
$$

It is easy to see that this gives us a bijection between two-line arrays and triples $\left(\pi, \Phi_{a}, \Phi_{b}\right)$ satisfying the above properties.

Applying the Schensted correspondence to the permutation $\pi$, we get a corresponding triple $\left((P, Q), \Phi_{a}, \Phi_{b}\right)$, where $P$ and $Q$ are standard Young tableaux of the same shape. From the properties of the insertion process for $K$ (cf. [12, Theorem 1], [18, Remarque 2]), Properties (1) and (2) translate to

(1') $\Phi_{a}(l+1)=\Phi_{a}(l)$ implies $i>i^{\prime}$ and $j \leqq j^{\prime}$, where $l, l+1$ occupy positions $(i, j),\left(i^{\prime}, j^{\prime}\right)$, respectively, in $Q$;

(2') $\Phi_{b}(l+1)=\Phi_{b}(l)$ implies $i>i^{\prime}$ and $j \leqq j^{\prime}$, where $l, l+1$ occupy positions $(i, j),\left(i^{\prime}, j^{\prime}\right)$, respectively, in $P$. Again, we have a bijection between such triples.

Now, given a triple $\left((P, Q), \Phi_{a}, \Phi_{b}\right)$, in which $P=\left(p_{i j}\right)$ and $Q=\left(q_{i j}\right)$ are standard Young tableaux of the same shape $\lambda, \sigma(\lambda)=N$, and $\Phi_{a}, \Phi_{b}$ are nondecreasing functions from $\{1,2, \cdots, N\}$ into the positive integers satisfying $\left(1^{\prime}\right)$ and $\left(2^{\prime}\right)$, we can define two new arrays $P^{\prime}=$ $\left(p_{i j}^{\prime}\right)$ and $Q^{\prime}=\left(q_{i j}^{\prime}\right)$ of shape $\lambda$ by letting $p_{i j}^{\prime}=\Phi_{b}\left(p_{i j}\right)$ and $q_{i j}^{\prime}=\Phi_{a}\left(q_{i j}\right)$ for all $(i, j)$ in $\Gamma(\lambda)$. The properties of $\Phi_{a}$ and $\Phi_{b}$ imply that $P^{\prime}$ and $Q^{\prime}$ are row-strict. It is not hard to show that every pair of row-strict plane partitions of the same shape is the unique image, under this map, of some triple $\left((P, Q), \Phi_{a}, \Phi_{b}\right)$.

Putting this chain of bijections together and applying $T$ to each of the row-strict plane partitions $P^{\prime}$ and $Q^{\prime}$, we have a bijection 
from Burge-type two-line arrays to pairs of column-strict plane partitions of the same shape. That this is Burge's correspondence follows directly by induction on the insertion process, noting that property (2) and the monotonicity of $\Phi_{b}$ implies that $\pi(k)$ replaces $\pi(l)$ if and only if $\Phi_{b}(\pi(k))$ replaces $\Phi_{b}(\pi(l))$.

In a similar fashion, $K$ can be factored through the Schensted correspondence.

The ability to factor $B$ and $K$ through Schensted's correspondence can facilitate in the proving of properties of the former correspondences. The property can be proved in the (usually) simpler form pertaining to Schensted's correspondence and then extended in the appropriate manner to $B$ or $K$. For example, in his work, Schensted demonstrated that his correspondence was a bijection between permutations and pairs of standard Young tableaux of the same shape. Theorem 3.1 follows immediately from this and the observations of this section. The use of this factorization in proofs will occur frequently in the remaining sections.

5. The Greene-type characterizations. The reason for Schensted's devising his correspondence was his interest in computing the lengths of the longest monotonic subsequences of a permutation. He showed that these lengths manifested themselves as the number of columns or rows in the corresponding tableaux. These results were extended by Greene to a characterization of the entire shape of the tableaux in terms of subsequences of the permutation.

Let $\pi$ be a permutation of the set $\{1,2, \cdots, n\}$. A $D$-subsequence of $\pi$ is a subset $V$ of $\{1,2, \cdots, n\}$ such that, if $i, j \in V$ with $i>j$, $\pi(i)>\pi(j)$. An A-subsequence is a subset $W$ such that if $i, j \in W$, $\pi(i)<\pi(j)$ whenever $i>j$. For $k \geqq 1$, let $d_{k}(\pi)$ be the maximum cardinality of the union of at most $k$ disjoint $D$-subsequences of $\pi$. Define $a_{k}(\pi)$ similarly, using $A$-subsequences.

TheOREm 5.1. (Greene [11].) Let $\pi$ be a permutation of $\{1,2$, $\cdots, n\}$, and let $\lambda$ be the shape of $K^{1}(\pi)$. Then, for $k \geqq 1$,

$$
\begin{aligned}
& d_{k}(\pi)=\lambda_{1}+\lambda_{2}+\cdots+\lambda_{k} \\
& a_{k}(\pi)=\lambda_{1}^{\prime}+\lambda_{2}^{\prime}+\cdots+\lambda_{k}^{\prime},
\end{aligned}
$$

where $\lambda^{\prime}$ is the conjugate shape of $\lambda$.

Using the factorization given in $\S 4$, we can apply this result to $B$ and $K$. We first must generalize the notion of monotonic subsequences. Let $M=\left(m_{i j}\right)$ be an $r \times c$ nonnegative integer matrix. A chain in $M$ is a sequence $\left(\left(i_{1}, j_{1}\right),\left(i_{2}, j_{2}\right), \cdots,\left(i_{l}, j_{l}\right)\right)$ of ordered pairs such that $1 \leqq i_{1} \leqq i_{2} \leqq \cdots \leqq i_{l} \leqq r, c \geqq j_{1} \geqq j_{2} \geqq \cdots \geqq j_{l} \geqq 1$ and 
the pair $(i, j)$ can occur at most $m_{i j}$ times in the sequence. A strict chain is a chain in which $1 \leqq i_{1}<i_{2}<\cdots<i_{l} \leqq r$ and $c \geqq j_{1}>$ $j_{2}>\cdots>j_{l} \geqq 1$. A cross chain in $M$ is a sequence $\left(\left(i_{1}, j_{1}\right),\left(i_{2}, j_{2}\right), \cdots\right.$, $\left.\left(i_{l}, j_{l}\right)\right)$ such that $v \geqq i_{1} \geqq i_{2} \geqq \cdots \geqq 1, c \geqq j_{1} \geqq j_{2} \geqq \cdots \geqq j_{l} \geqq 1$, and $(i, j)$ can occur at most $m_{i j}$ times. A strict cross chain is a cross chain in which $r \geqq i_{1}>i_{2}>\cdots>i_{l} \geqq 1$ and $c \geqq j_{1}>j_{2}>\cdots>j_{l} \geqq 1$. We allow empty chains and cross chains, strict or otherwise.

For $k \geqq 1$, let $a_{k}(M)=\max \left\{\left|V_{1}\right|+\cdots+\left|V_{k}\right|: V_{l}\right.$ are chains such that the number of times $(i, j)$ appears in all of them combined is at most $\left.m_{i j}\right\}$. We define $\widetilde{d}_{k}(M), d_{k}(M)$ and $\widetilde{\alpha}_{k}(M)$ by replacing "chains" in the definition of $a_{k}(M)$ by "strict cross chains," "cross chains" and "strict chains," respectively. Note that for all $k \geqq \min \{r, c\}$, we have $a_{k}(M)=d_{k}(M)=\sigma(M)$.

As an illustration of these definitions, consider the matrix

$$
M=\left(\begin{array}{llll}
1 & 0 & 1 & 1 \\
0 & 2 & 2 & 0 \\
1 & 0 & 1 & 0
\end{array}\right)
$$

The longest chain is $((1,4),(1,3),(2,3),(2,3),(2,2),(2,2),(3,1))$; the longest cross chain is $((3,3),(2,3),(2,3),(2,2),(2,2),(1,1))$. Thus, $a_{1}(M)=7$ and $d_{1}(M)=6$. The remaining values of $a_{k}$ and $d_{k}$, as well as all the values of $\widetilde{a}_{k}$ and $\widetilde{d}_{k}$, for the given matrix are exhibited in the table below.

\begin{tabular}{rcccc}
\hline$k$ & $a_{k}$ & $\widetilde{a}_{k}$ & $d_{k}$ & $\widetilde{d}_{k}$ \\
\hline 1 & 7 & 3 & 6 & 3 \\
2 & 8 & 5 & 8 & 4 \\
3 & 9 & 6 & 9 & 5 \\
4 & 9 & 7 & 9 & 6 \\
5 & 9 & 8 & 9 & 7 \\
6 & 9 & 9 & 9 & 8 \\
$\geqq 7$ & 9 & 9 & 9 & 9 \\
\hline
\end{tabular}

With these definitions, we can now interpret Greene's theorem in terms of $B$ and $K$.

THEOREM 5.2. Let $M$ be a nonnegative integer matrix. Let $\lambda$ and $\gamma$ be the shapes of $B^{1}(M)$ and $K^{1}(M)$, respectively. Let $\lambda^{\prime}$ and. $\gamma^{\prime}$ be the conjugate shapes. Then, for $k \geqq 1$,

(i) $a_{k}(M)=\lambda_{1}+\cdots+\lambda_{k}$.

(ii) $\widetilde{d}_{k}(M)=\lambda_{1}^{\prime}+\cdots+\lambda_{k}^{\prime}$.

(iii) $d_{k}(M)=\gamma_{1}+\cdots+\gamma_{k}$.

(iv) $\widetilde{a}_{k}(M)=\gamma_{1}^{\prime}+\cdots+\gamma_{k}^{\prime}$. 
Proof. We consider parts (i) and (ii), the analysis for (iii) and (iv) being similar. Suppose that, under Burge's correspondence, $M$ is associated with the two-line array $\left(\begin{array}{llll}a_{1} & a_{2} & \cdots & a_{n} \\ b_{1} & b_{2} & \cdots & b_{n}\end{array}\right)$. Note that chains and strict cross chains in $M$ correspond to nondecreasing subsequences and strictly decreasing subsequences, respectively, of $\left(b_{1}, b_{2}, \cdots, b_{n}\right)$.

If we factor $B$ through the Schensted map, and obtain a permutation $\pi$ associated with the two-line array of $M$, we see that the nondecreasing and decreasing subsequences of $\left(b_{1}, b_{2}, \cdots, b_{n}\right)$ correspond precisely to $A$-subsequences and $D$-subsequences of $\pi$. From this, it follows immediately that $a_{k}(M)=a_{k}(\pi)$ and $\widetilde{d}_{k}(M)=d_{k}(\pi)$.

The factorization also tells us that $\lambda$ is the shape of $T K^{1}(\pi)$. Hence, by Theorem 5.1, we have $a_{k}(\pi)=\lambda_{1}+\cdots+\lambda_{k}$ and $d_{k}(\pi)=$ $\lambda_{1}^{\prime}+\cdots+\lambda_{k}^{\prime}$, giving us (i) and (ii).

Theorem 5.2 suggests a manner in which to characterize not just the shape of $B^{1}(M)$ or $K^{1}(M)$, but all of $B(M)$ or $K(M)$ in terms of chains and cross chains in $M$. To achieve this, we first need the following result.

LEMmA 5.3. Let $h \geqq 0$, and let $\beta=\left(b_{1}, b_{2}, \cdots, b_{n}\right)$ be a sequence of positive integers. Let $P=B^{1}(\beta)$ and $\bar{P}=K^{1}(\beta)$. Let $\beta^{\prime}$ be the sequence derived from $\beta$ by deleting those terms less than or equal to $h$. Let $P^{\prime}$ and $\bar{P}^{\prime}$ be the column-strict plane partitions derived from $P$ and $\bar{P}$, respectively, by deleting those parts less than or equal to $h$. Then

(i) $P^{\prime}=B^{1}\left(\beta^{\prime}\right)$.

(ii) $\bar{P}^{\prime}=K^{1}\left(\beta^{\prime}\right)$.

Proof. The lemma is easily proven by induction on $n$. Let $\widetilde{\beta}=\left(b_{1}, b_{2}, \cdots, b_{n}, b_{n+1}\right), \widetilde{P}=B^{1}(\widetilde{\beta})$ and let $\widetilde{\beta}^{\prime}$ and $\widetilde{P}^{\prime}$ be the corresponding derived sequence and column-strict plane partition. By induction, we have $P^{\prime}=B^{1}\left(\beta^{\prime}\right)$. If $b_{n+1} \leqq h, \beta^{\prime}=\widetilde{\beta}^{\prime}$. In addition, when $b_{n+1}$ is inserted into $P$, it does not affect any part greater than $h$, implying that $P^{\prime}=\widetilde{P}^{\prime}$. Thus, $\widetilde{P}^{\prime}=P^{\prime}=B^{1}(\beta)=B^{1}\left(\widetilde{\beta}^{\prime}\right)$.

On the other hand, if $b_{n+1}>h$, when it is inserted into $P$, the process only involves parts greater than $h$, exactly as though $b_{n+1}$ were inserted into $P^{\prime}$, until a part is placed at the end of a column or a part less than or equal to $h$ is replaced, after which, no part of $P$ greater than $h$ is ever again affected. Thus, inserting $b_{n+1}$ into $P$ affects the parts greater than $h$ in the same manner as inserting $b_{n+1}$ into $P^{\prime}$. Since $P^{\prime}=B^{1}\left(\beta^{\prime}\right)$, we have $\widetilde{P}^{\prime}=B_{b_{n+1}} P^{\prime}=B^{1}\left(\widetilde{\beta}^{\prime}\right)$.

The proof of (ii) is exactly the same if we use " $K$ " instead of " $B$ " and "row" instead of "column". 
This lemma tells us how to divide the column-strict plane partition into layers. We can then employ Theorem 5.2 on each of the layers to get the desired characterization. If $M=\left(m_{i j}\right)$ is an $r \times c$ matrix and $l$ is an integer, $1-r \leqq l \leqq c-1$, 'define $M_{l}$ to be the $r \times c$ matrix obtained from $M$ by changing the entries in the first $l$ columns $\left(-l\right.$ rows) to zero if $l>0(l<0)$. If $l=0$, let $M_{0}=M$.

THEOREM 5.4. Let $M$ be a nonnegative integer matrix of size $r \times c$, and let $1-r \leqq l \leqq c-1$. Let $B(M)=(P, Q)$, where $P=\left(p_{i j}\right)$ and $Q=\left(q_{i j}\right)$, and let $K(M)=(\bar{P}, \bar{Q})$, where $\bar{P}=\left(\bar{p}_{i j}\right)$ and $\bar{Q}=\left(\bar{q}_{i j}\right)$. We define two shapes $\lambda$ and $\gamma$ by

$$
\begin{aligned}
\lambda_{i} & = \begin{cases}\left\{\left\{k: q_{i k} \geqq l+1\right\} \mid\right. & \text { if } l \geqq 0 \\
\left|\left\{k: p_{i k} \geqq 1-l\right\}\right| & \text { if } l \leqq 0\end{cases} \\
\gamma_{i} & = \begin{cases}\left|\left\{k: \bar{q}_{i k} \geqq l+1\right\}\right| & \text { if } l \geqq 0 \\
\left|\left\{k: \bar{p}_{i k} \geqq 1-l\right\}\right| & \text { if } l \leqq 0 .\end{cases}
\end{aligned}
$$

Let $\lambda^{\prime}$ and $\gamma^{\prime}$ be the respective conjugates of $\lambda$ and $\gamma$. Then, for $k \geqq 1$,
(i ) $a_{l k}\left(M_{l}\right)=\lambda_{1}+\cdots+\lambda_{l}$.
(ii) $\widetilde{d}_{k}\left(M_{l}\right)=\lambda_{1}^{\prime}+\cdots+\lambda_{k}^{\prime}$.
(iii) $d_{k}\left(M_{l}\right)=\gamma_{1}+\cdots+\gamma_{k}$.
(iv) $\widetilde{a}_{k}\left(M_{l}\right)=\gamma_{1}^{\prime}+\cdots+\gamma_{k}^{\prime}$.

Proof. We will prove (i), the proof of (iii) being similar. Parts (ii) and (iv) then follow from Theorem 5.2. Considering Burge's correspondence, let $M$ correspond to the two-line array

$$
\left(\begin{array}{llll}
a_{1} & a_{2} & \cdots & a_{N} \\
b_{1} & b_{2} & \cdots & b_{N}
\end{array}\right)
$$

If $l \geqq 0$, let $T=\max \left\{i: a_{i} \geqq l+1\right\}$. Then $M_{l}$ corresponds to the two-line array

$$
\left(\begin{array}{llll}
a_{1} & a_{2} & \cdots & a_{T} \\
b_{1} & b_{2} & \cdots & b_{T}
\end{array}\right)
$$

It follows immediately from the construction that the shape of $B^{1}\left(b_{1}, b_{2}, \cdots, b_{T}\right)$ is $\lambda$. But by Theorem 5.2 , the shape of $B^{1}\left(b_{1}, b_{2}, \cdots, b_{T}\right)$ is $\left(a_{1}\left(M_{l}\right), a_{2}\left(M_{l}\right)-a_{1}\left(M_{l}\right), a_{3}\left(M_{l}\right)-a_{2}\left(M_{l}\right), \cdots\right)$. This implies (i) for $l \geqq 0$.

If $l \leqq 0$, the two-line array of $M_{l}$ is obtained by deleting from the two-line array of $M$ all columns of the form $\left(\begin{array}{l}a \\ b\end{array}\right)$ where $b \leqq-l$. Let $\beta=\left(b_{1}, b_{2}, \cdots\right)$. Then, using the notation and the result of Lemma 5.3 with $h=-l$, we have $B^{1}\left(\beta^{\prime}\right)=P^{\prime}$, implying that the 
shape of $B^{1}\left(\beta^{\prime}\right)$ is $\lambda$. But $\beta^{\prime}$ is the bottom row of the two-line array of $M_{l}$. So, by Theorem 5.2, the shape of $B^{1}\left(\beta^{\prime}\right)$ is $\left(a_{1}\left(M_{l}\right), a_{2}\left(M_{l}\right)-\right.$ $\left.a_{1}\left(M_{l}\right), \cdots\right)$, completing the proof.

We note that the original difference in the constructions of $P$ and $Q$ is missing from this theorem. The theorem can be reformulated in terms of plane partitions, in which form it will relate $B$ to another plane partition correspondence $(\S 10)$.

THEOREM 5.5. Let $M$ be a nonnegative integer matrix of size $r \times c$ and let $l$ be an integer, $1-r \leqq l \leqq c-1$. Considering the plane partitions $A=B(M)$ and $\bar{A}=K(M)$, we let $\lambda$ and $\gamma$ be the ldiagonals of $A$ and $\bar{A}$, respectively. Let $\lambda^{\prime}$ and $\gamma^{\prime}$ be their respective conjugates. Then, for $k \geqq 1$,

(i ) $a_{k}\left(M_{l}\right)=\lambda_{1}+\cdots+\lambda_{k}$.

(ii) $\widetilde{d}_{k}\left(M_{l}\right)=\lambda_{1}^{\prime}+\cdots+\lambda_{k}^{\prime}$.

(iii) $d_{k}\left(M_{l}\right)=\gamma_{1}+\cdots+\gamma_{k}$.

(iv) $\tilde{a}_{k}\left(M_{l}\right)=\gamma_{1}^{\prime}+\cdots+\gamma_{k}^{\prime}$.

Proof. If $B(M)=(P, Q)$, where $P=\left(p_{i j}\right)$ and $Q=\left(q_{i j}\right)$ are column-strict plane partitions of the same shape, $A$ is obtained by applying the Frobenius construction to $(P, Q)$. Thus,

$$
\lambda_{i}= \begin{cases}\left|\left\{k: q_{i k} \geqq l+1\right\}\right| & \text { if } l \geqq 0 \\ \left|\left\{k: p_{i k} \geqq 1-l\right\}\right| & \text { if } l \leqq 0\end{cases}
$$

(cf. $\S 2$ ). Comparing this with Theorem 5.4, (i) and (ii) follow. The proof of (iii) and (iv) is analogous.

Theorem 5.4 and its more elegant form Theorem 5.5 completely characterize the correspondences $B$ and $K$. Surprisingly, considering the original constructions, these theorems imply that the correspondences preserve symmetry. We note that $(T M)_{l}=T\left(M_{-l}\right)$. Therefore, $a_{k}\left((T M)_{l}\right)=a_{k}\left(T\left(M_{-l}\right)\right)=a_{k}\left(M_{-l}\right)$. The same equality holds for $d_{k}$. From these observations, we obtain the following results.

CoRollary 5.6. (i ) $B^{1}(M)=B^{2}(T M)$ or, as plane partitions, $B(T M)=T B(M)$.

(ii) $K^{1}(M)=K^{2}(T M)$ or, as plane partitions, $K(T M)=$ $T K(M)$.

CoROLlary 5.7. $B$ and $K$ are bijections between symmetric nonnegative integer matrices and column-strict plane partitions (equivalently, symmetric plane partitions). 
These corollaries were first proved by Schützenberger [21] in terms of Schensted's correspondence, and later by Burge and Knuth in terms of column-strict plane partitions.

If $M$ is a symmetric matrix, we have seen that $B^{1}(M)=B^{2}(M)$ and $K^{1}(M)=K^{2}(M)$. In this case, the correspondences possess another pair of interesting and useful [4] properties. It turns out that the trace of $M$ equals the number of odd length columns in $K^{1}(M)$, and the number of odd entries on the main diagonal of $M$ equals the number of odd length rows in $B^{1}(M)$. These are observations of Knuth and Burge, respectively. One can find in [15] a proof of the former, which in turn can be revised to yield a proof of the latter (cf. [4]). A proof of the latter can also be obtained by restricting the former to Schensted's correspondence and then utilizing the factorization of $B$ through that correspondence.

6. Some properties of $B^{1}$ and $K^{1}$. The functions $B^{1}$ and $K^{1}$ can be viewed as maps from finite sequences of positive integers to column-strict plane partitions. We would now like to consider some of their properties, especially concerning the effect of modifying the sequence.

It is an immediate corollary of Theorem 3.1 that $B^{1}$ and $K^{1}$ are not one-to-one. This leads one to attempt to characterize those sequences that are the preimages of some fixed column-strict plane partition. This task was accomplished for both $K^{1}$ and $B^{1}$ by Knuth [15]. We give his result below.

Two sequences of positive integers are called K-equivalent if one can be transformed into another by a series of interchanges of the following types:

(i) $(\cdots, a, c, b, \cdots) \leftrightarrow(\cdots, c, a, b, \cdots)$ where $a<b \leqq c$.

(ii) $(\cdots, b, a, c, \cdots) \leftrightarrow(\cdots, b, c, a, \cdots)$ where $a \leqq b<c$.

Two sequences are B-equivalent if one can be transformed into the other by a series of interchanges of the following types:

(i) $(\cdots, a, c, b, \cdots) \leftrightarrow(\cdots, c, a, b, \cdots)$ where $a \leqq b<c$.

(ii) $(\cdots, b, a, c, \cdots) \leftrightarrow(\cdots, b, c, a, \cdots)$ where $a<b \leqq c$.

THEOREM 6.1. Let $\alpha$ and $\beta$ be finite sequences of positive integers. Then

(i) $K^{1}(\alpha)=K^{1}(\beta)$ if and only if $\alpha$ and $\beta$ are $K$-equivalent.

(ii) $B^{1}(\alpha)=B^{1}(\beta)$ if and only if $\alpha$ and $\beta$ are B-equivalent.

This theorem can be a useful tool in obtaining results concerning $B$ and $K$. One shows that $B$ or $K$ has a certain property acting on a canonical sequence in each equivalence class, and then that the property is not affected by interchanges. Greene proved Theorem 
5.1 in this fashion (also Theorem 3 in [12]), and the technique can also be used to demonstrate the next theorem. However, with Theorem 5.4 at our disposal the proof becomes trivial.

THEOREM 6.2. Let $\beta=\left(b_{1}, b_{2}, \cdots, b_{c}\right)$ be a sequence of positive integers. Let $\bar{\beta}=\left(b_{c}, \cdots, b_{2}, b_{1}\right)$. Then $B^{1}(\beta)=K(\bar{\beta})$.

Proof. Let $r=\max \left\{b_{1}, b_{2}, \cdots, b_{c}\right\}$. Let $M$ be the $r \times c$ matrix

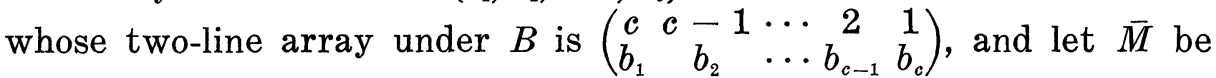
the $r \times c$ matrix whose two-line array under $K$ is $\left(\begin{array}{ccccc}c & c-1 & \cdots & 2 & 1 \\ b_{c} & b_{c-1} & \cdots & b_{2} & b_{1}\end{array}\right)$. Then $\bar{M}$ is $M$ reflected about a vertical axis and it follows immediately from Theorem 5.4 that $B^{1}(M)=K^{1}(\bar{M})$.

This result can be found in a variety of forms in the literature, e.g., [16, p. 59] or [17, Theorem 4.5]. It was originally proved in terms of permutations by Schensted [20], using induction and a form of the following corollary.

COROLlARY 6.3. Let $P$ be a column-strict plane partition, and let $x$ and $y$ be positive integers. Then $B_{x} K_{y} P=K_{y} B_{x} P$.

Proof. Let $\left(b_{1}, b_{2}, \cdots, b_{n}\right)$ be a sequence of positive integers such that $P=B^{1}\left(b_{1}, \cdots, b_{n}\right)$. By repeated applications of Theorem 6.2, one can easily verify that

$$
K_{y} B_{x} P=B^{1}\left(y, b_{1}, \cdots, b_{n}, x\right)=B_{x} K_{y} P,
$$

thereby completing the proof.

Theorem 6.2 and its corollary can be useful in deriving other properties of $B, K$ and related correspondences (cf. [24], [25]). They also lead us to another connection between $B$ and $K$, which we explore in the next two sections.

7. The operations of Schützenberger. Suppose that the image of a two-line array under the Burge correspondence is $(P, Q)$ whereas the image of the same array with its first column deleted is $\left(P^{\prime}, Q^{\prime}\right)$. Theorem 6.2 tells us that, if we know the difference in the shapes of $P^{\prime}$ and $P$, we can obtain $P^{\prime}$ and $b_{1}$ from $P$ by reversing the Knuth construction.

For example, if

$$
\begin{array}{rrrr}
4 & 4 & 3 & 2 \\
3 & 2 & 2 \\
2 & 1 & 1
\end{array}
$$


and the shape of $P^{\prime}$ is $(4,3,2)$, we must have $b_{1}=3$ and

$$
\begin{array}{rrrr}
4 & 4 & 2 & 2 \\
P^{\prime}= & 2 & 1 \\
2 & 1 & &
\end{array},
$$

as these are the unique choices of $b_{1}$ and a column-strict plane partition $P^{\prime}$ of shape $(4,3,2)$ such that $K_{b_{1}} P^{\prime}=P$. The same result holds for Knuth's correspondence by reversing the roles of $B$ and $K$.

To obtain the relation between $Q$ and $Q^{\prime}$, we have need of an operation $\Delta$ on column-strict plane partitions due to Schützenberger [21]. It will turn out that $Q^{\prime}=\Delta Q$. To construct $\Delta Q$, where $Q=\left(q_{i j}\right)$, start by deleting $q_{11}$. This creates a "hole" which we fill using the procedure described below. This creates another hole, which again we fill by the same procedure. This process continues until we can no longer fill the hole, at which point we have a new column-strict plane partition which we denote by $\Delta Q$.

If we have a "hole" at position $(i, j)$ in $Q$, we fill it by $q_{i j+1}$, creating a new hole at position $(i, j+1)$, if $q_{i j+1}>q_{i+1 j}$. Otherwise, we fill the hole with $q_{i+1 j}$, creating a new hole at the $(i+1, j)$ position. We use the convention that $q_{k l}=0$ if $(k, l)$ is not in $\Gamma(\lambda)$.

Illustrations of this operation can be found in [8], [16, pp. 5758], [21] or [22].

Concerning standard Young tableaux and Schensted's correspondence, Schützenberger proved the following property of $\Delta$.

TheOREM 7.1. Let $\pi$ be a permutation on $\{1,2, \cdots, n+1\}$. Define a permutation $\pi^{\prime}$ on $\{1,2, \cdots, n\}$ by letting

$$
\pi^{\prime}(i)= \begin{cases}\pi(i)-1 & \text { if } \pi(i)>\pi(n+1) \\ \pi(i) & \text { if } \pi(i)<\pi(n+1) .\end{cases}
$$

Then $K^{2}\left(\pi^{\prime}\right)=\Delta K^{2}(\pi)$.

To extend this result to $B$ and $K$, we factor them through the Schensted correspondence. In the case of $B$, if we have a two-line array

$$
\left(\begin{array}{llll}
a_{1} & a_{2} & \cdots & a_{n+1} \\
b_{1} & b_{2} & \cdots & b_{n+1}
\end{array}\right)
$$

to which we can apply $B$, it has an associated permutation $\pi$ and functions $\Phi_{a}$ and $\Phi_{b}$ (cf. $\S 4$ ). We can obtain $B^{2}$ of this array by applying $\Phi_{a}$ to each part of the transpose of $K^{2}(\pi)$.

From the construction of the factorization, we know that $\Phi_{a}(j)=$ $\Phi_{a}(j+1)$ if and only if $j$ appears in the same column or to the left 
of, and below, $j+1$ in $K^{2}(\pi)$. This implies that $J$ "commutes" with $\Phi_{a}$, i.e., it makes no difference whether we apply $\Delta$ to $\Phi_{a}\left(T K^{2}(\pi)\right)$, or first apply $\Delta$ to $T K^{2}(\pi)$ and then apply $\Phi_{a}$.

In addition, if the array

$$
\left(\begin{array}{lll}
a_{2} & \cdots & a_{n+1} \\
b_{2} & \cdots & b_{n+1}
\end{array}\right)
$$

is associated (as in $\S 4$ ) with the permutation $\pi^{\prime}$ and the functions $\Phi_{a}^{\prime}$ and $\Phi_{b}^{\prime}$, it is clear that $\pi$ and $\pi^{\prime}$ are related as in Theorem 7.1 and that $\Phi_{a}^{\prime}$ is the restriction of $\Phi_{a}$ to $\{1,2, \cdots, n\}$.

A similar collection of facts holds for $K$. If we add them all to Theorem 7.1, we obtain the desired extension.

COROLLARY 7.2. (i ) $B^{2}\left(\begin{array}{lll}a_{2} \cdots & a_{n} \\ b_{2} & \cdots & b_{n}\end{array}\right)=\Delta B^{2}\left(\begin{array}{llll}a_{1} & a_{2} & \cdots & a_{n} \\ b_{1} & b_{2} & \cdots & b_{n}\end{array}\right)$.

(ii) $K^{2}\left(\begin{array}{lll}a_{2} & \cdots & a_{n} \\ b_{2} & \cdots & b_{n}\end{array}\right)=\Delta K^{2}\left(\begin{array}{llll}a_{1} & a_{2} & \cdots & a_{n} \\ b_{1} & b_{2} & \cdots & b_{n}\end{array}\right)$.

Using $\Delta$, we can define a collection of operators, essentially defined by Schützenberger, each of which maps one column-strict plane partition into another, preserving shapes. Choose $m \geqq 1$, and let $P$ be a column-strict plane partition with largest part at most $m$. We inductively construct a new array $S_{m}(P)=\left(p_{i j}^{\prime}\right)$ based on $P$ and having the same shape as $P$.

Start by letting $P_{1}=P$. If, at some point, we have defined $P_{l}, l \geqq 1$, let $n_{l}$ be the size of the largest part in $P_{l}$ and let $\left(i_{l}, j_{l}\right)=$ $\Gamma(\lambda)-\Gamma(\tau)$, where $\lambda$ and $\tau$ are the shapes of $P_{l}$ and $\Delta P_{l}$, respectively. We then define $p_{i_{l} j_{l}}^{\prime}=m-n_{l}+1$. If $\Delta P_{l}$ is not empty, let $P_{l+1}=\Delta P_{l}$ and continue the process. When $\Delta P_{l}$ is empty, the construction is complete.

Thus, if we have the column-strict plane partition

$$
\begin{array}{rrrr}
4 & 4 & 4 & 3 \\
3 & 3 & 1 & \\
1 & 1 &
\end{array}
$$

we find that

$$
\begin{array}{rlll}
S_{5}(P)= & 5 & 5 & 2 \\
3 & 3 & 3 & \\
2 & 2 & &
\end{array} \text {. }
$$

For our purposes, we are mainly interested in the following property of $S_{m}$.

TheOREM 7.3. For $m \geqq 1, S_{m}$ is an involution mapping the set 
of column-strict plane partitions with part size bounded by $m$ onto itself, preserving shapes.

A proof of this theorem is given in [8], along with an extension of the definition of $S_{m}$ and a discussion of some of its other properties. Variations of $S_{m}$ are also considered in [16, pp. 57-60] and [22].

8. The dihedral group and the correspondences. Some of the results that we have derived thus far have suggested that there is a relation between geometric operations on a matrix and changes in the image of the matrix under $B$ and $K$. The most obvious relation is the fact that $B$ and $K$ commute with $T$. In addition, Theorem 5.4 implies that, if we take a matrix $M$ and let $\bar{M}$ be the reflection of $M$ about a vertical axis, we have $B^{1}(M)=K^{1}(\bar{M})$ (cf. Theorem 6.2).

We are thus led to consider the effect of the eight operations, forming the dihedral group generated by $90^{\circ}$ rotations and transpositions, on a matrix under $B$ and $K$. If $M=\left(m_{i j}\right)$ is an $r \times c$ matrix, let $U M=\left(m_{i j}^{\prime}\right)$ be the $c \times r$ matrix defined by $m_{i j}^{\prime}=m_{r-j+1 i}$. Thus $U$ rotates a matrix $90^{\circ}$ clockwise. As Corollary 5.6 tells us the effect of the transposition $T$, it suffices to determine how $K$ and $B$ are affected by $U$.

THEOREM 8.1. Let $M$ be a $r \times$ c matrix. Then

(i ) $B(U M)=\left(K^{2}(M), S_{r} K^{1}(M)\right)$.

(ii) $K(U M)=\left(B^{2}(M), S_{r} B^{1}(M)\right)$.

Proof. We first prove (i). That $B^{1}(U M)=K^{2}(M)$ for all $M$ is an immediate consequence of Theorem 5.4. We next prove that $B^{2}(U M)=S_{r} K^{2}(T M)$ which, since $K^{2}(T M)=K^{1}(M)$, will finish the proof. Since $K^{2}(M)=K^{1}(T M), B^{2}(U M)$ has the same shape as $S_{r} K^{2}(T M)$ for all $M$. Now, the desired equality can be proved by induction on $\sigma(M)$, the case $\sigma(M)=1$ being clear.

Let $M=\left(m_{i j}\right)$ be an $r \times c$ matrix. Let $k$ be the greatest index such that row $k$ of $M$ has nonzero entries, and let $l$ be the greatest index such that $m_{k l} \neq 0$. Define a new $r \times c$ matrix $M^{\prime}$ by subtracting 1 from $m_{k l}$ in $M$.

Using the Burge correspondence, it is clear that the two-line array of $U M^{\prime}$ can be obtained by deleting the last column, necessarily $\left(\begin{array}{c}r-k+1 \\ l\end{array}\right)$, from the two-line array of $U M$. Thus, we can construct $B^{2}(U M)$ by adjoining $r-k+1$ to some position $(s, t)$ in $B^{2}\left(U M^{\prime}\right)$.

On the other hand, using the Knuth correspondence, the twoline array of $T M^{\prime}$ equals the two-line array of $T M$ with its first 
column, necessary $\left(\begin{array}{l}k \\ l\end{array}\right)$, deleted. Thus by Corollary 7.2, $S_{r} K^{2}\left(T M^{\prime}\right)=$ $S_{r} \Delta K^{2}(T M)$. The definition of $S_{r}$ implies that, to get $S_{r} K^{2}(T M)$, we adjoin $r-k+1$ to some position $\left(s^{\prime}, t^{\prime}\right)$ in $S_{r} K^{2}\left(T M^{\prime}\right)$. By induction, $B^{2}\left(U M^{\prime}\right)=S_{r} K^{2}\left(T M^{\prime}\right)$ and, since $B^{2}(U M)$ and $S_{r} K^{2}(T M)$ have the same shape, $(s, t)=\left(s^{\prime}, t^{\prime}\right)$. Hence, $B^{2}(U M)=S_{r} K^{2}(T M)$.

The proof of (ii) is now easy. It follows from Theorem 5.4 that $B^{2}(M)=K^{1}(U M)$ and $K^{2}(M)=B^{2}(T U M)$. From the second of these and (i), we obtain

$$
K^{2}(U M)=B^{2}\left(U^{2} T M\right)=S_{r} K^{1}(U T M) .
$$

Using the first equality and Corollary 5.6, we find that

$$
S_{r} K^{1}(U T M)=S_{r} B^{2}(T M)=S_{r} B^{1}(M),
$$

completing the proof.

This result and Corollary 5.6 allow us to calculate how $B$ and $K$ act upon any of the eight variants of the $r \times c$ matrix $M$ using just $S_{r}$ and $S_{c}$ if we are given $B(M)$ and $K(M)$. For example, if $K(M)=(P, Q)$, we find that $K\left(U^{2} M\right)$ equals $\left(S_{r} P, S_{c} Q\right)$. Note that the fact that $S_{r}$ is an involution (Theorem 7.3) is an immediate consequence of this result, since $U^{2}\left(U^{2} M\right)=M$.

If we restrict ourselves to Schensted's correspondence and let $M$ be a permutation matrix, Theorem 8.1 becomes simpler, since $K^{1}(M)=T B^{1}(M)$ and $K^{2}(M)=T B^{2}(M)$. In this case, the corollary is basically a reformulation of results of Schensted and Schützenberger. These results and some of their implications are given in another form by Knuth [16, p. 59].

9. The Hillman-Grassl correspondence. Although the study of plane partitions dates back to MacMahon, reverse plane partitions have only recently been considered. Interestingly, reverse plane partitions are much easier to enumerate than their forward cousins. Using some deep results concerning plane partitions, Stanley has shown that reverse plane partitions have a simple generating function (cf. [23, p. 270]). This simplicity was explained by a correspondence between nonnegative integer arrays and reverse plane partitions devised by Hillman and Grassl [13]. It is to this correspondence that we turn our attention in this section.

Let $\lambda$ be a shape. Let $M=\left(m_{i j}\right)$ be an array of nonnegative integers indexed by $\Gamma(\lambda)$. We say that $M$ has shape $\lambda$. We will use $M$ to construct inductively a reverse plane partition $H(M)$ of shape $\lambda$. If all the entries of $M$ are 0 , we let $H(M)$ be the reverse plane partition of shape $\lambda$ with all of its parts equal to 0 . 
If $M$ has some nonzero entry, let $t=\min \left\{j: m_{\imath j} \neq 0\right.$ for some $\left.i\right\}$ and let $s=\max \left\{i: m_{i t} \neq 0\right\}$. Let $M^{\prime}$ be the array of shape $\lambda$ obtained from $M$ by subtracting 1 from $m_{s t}$. We assume that $H\left(M^{\prime}\right)=\left(q_{i j}\right)$ has been defined.

We next define a path in $\Gamma(\lambda)$. Let $\left(i_{1}, j_{1}\right)=\left(s, \lambda_{s}\right)$. Assume that $\left(i_{k}, j_{k}\right)$ has been defined for some $k \geqq 1$. Let $\left(i_{k+1}, j_{k+1}\right)$ be $\left(i_{k}+1, j_{k}\right)$ if $q_{i_{k} j_{k}}=q_{i_{k}+1 j_{k}}$ and let $\left(i_{k+1}, j_{k+1}\right)$ be $\left(i_{k}, j_{k}-1\right)$ otherwise. By convention, we assume that $q_{i j}=\infty$ if $(i, j)$ is not in $\Gamma(\lambda)$. It is easily seen that we must have $\left(i_{n}, j_{n}\right)=\left(\lambda_{t}^{\prime}, t\right)$ for some $n$. The path terminates in this position.

Finally, we let $H(M)$ be the array $\left(p_{\imath j}\right)$ of shape $\lambda$ defined by

$$
p_{i j}= \begin{cases}q_{2 j}+1 & \text { if }(i, j)=\left(i_{k}, j_{k}\right) \text { for some } k \\ q_{2 j} & \text { otherwise }\end{cases}
$$

for all $(i, j)$ in $\Gamma(\lambda)$. Detailed examples of this procedure can be found in [9] or [13].

The map $H$ is the Hillman-Grassl correspondence. The fundamental result concerning $H$ is given below.

THEOREM 9.1. For a given shape $\lambda, H$ is a bijection from the set of all nonnegative integer arrays of shape $\lambda$ onto the set of all reverse plane partitions of shape $\lambda$.

A proof of this theorem can be found in [13], along with certain other properties of $H$. Additional properties of the correspondence as it pertains to reverse plane partitions are given in [9] and [14]. Here, we are interested in a connection between $H$ and the plane partition correspondences that we have already developed.

10. The relationship between $B$ and $H$. Despite the entirely different constructions used to define the correspondences $B$ and $H$, it happens that they are intimately connected. Indeed, $H$ can be viewed as a generalization of $B$, and it can be used to give a simpler construction of $B(M)$. To see this connection, we first note that the Hillman-Grassl correspondence possesses a very familiar type of characterization.

THEOREM 10.1. Let $M$ be a nonnegative integer array of shape $\tau$. Choose $l$ such that $1-\rho(\tau) \leqq l \leqq \tau_{1}-1$. Let $s=\max \{i:(i, l+i) \in$ $\Gamma(\tau)\}$ and let $t=l+s$. Define a matrix $M^{l}$ to be the $s \times t$ subarray of $M$ consisting of those entries in positions $(i, j), 1 \leqq i \leqq s, 1 \leqq j \leqq t$.

Let $\lambda$ be the shape determined by the l-diagonal of $H(M)$ and let $\lambda^{\prime}$ be its conjugate shape. Then, for all $k \geqq 1$, we have 
(i) $a_{k}\left(M^{l}\right)=\lambda_{1}+\lambda_{2}+\cdots+\lambda_{k}$
(ii) $\tilde{d}_{k}\left(M^{l}\right)=\lambda_{1}^{\prime}+\lambda_{2}^{\prime}+\cdots+\lambda_{k}^{\prime}$.

Proof. A direct proof of part (i) can be found in [14]. This can also be proved by first verifying Theorem 10.2, to follow, invoking Theorem 5.5 and then noting that, to determine a diagonal in the general case, one can restrict oneself to the rectangular case. The details for this method are given in [7, pp. 110-121].

As for part (ii), this follows immediately from part (i) and Theorem 5.2.

This theorem suggests a strong relationship between $H$ and the Burge correspondence. To make the connection explicit, consider the following correspondence. Let $M$ be an $r \times c$ nonnegative integer matrix. Rotate $M 180^{\circ}$, apply $H$ to the rotated matrix to yield a reverse plane partition of shape $\left(c^{r}\right)$ and then rotate the reverse plane partition $180^{\circ}$ to yield a plane partition with at most $r$ rows and $c$ columns. We denote this resultant plane partition by $G(M)$. Thus, $G$ sends an $r \times c$ matrix to a plane partition with at most $r$ rows and $c$ columns, as does $B$. From the definition of $G$ and the nature of $H, G$ must be a bijection, as is $B$. Finally, a comparison of Theorems 5.5 and 10.1 leads us the following conclusion.

\section{THEOREM 10.2. For all nonnegative integer matrices $M, B(M)=$ $G(M)$.}

A direct proof of this theorem can be found in [7, pp. 110-118]. This is a surprising result. We have no good explanation as to why this connection should exist. Nor do we know of any analogous, and equally nice, result for $K$. Each step in the construction of $K(M)$ does consist of adding 1's to certain parts, but these parts need not be contiguous, forming a path, and the first and last positions are not easy to determine. For another example of where the parallelism of $B$ and $K$ seems to fail, see $[10, \S 6]$.

\section{REFERENCES}

1. G. E. Andrews, The Theory of Partitions, Addison-Wesley, Reading, Mass., 1976.

2. E. A. Bender and D. E. Knuth, Enumeration of plane partitions, J. Combinatorial Theory, Ser. A, 13 (1972), 40-54.

3. G. Birkhoff, Lattice Theory, 3rd. ed., Amer. Math. Soc., Providence, RI, 1967.

4. W. H. Burge, Four correspondences between graphs and generalized Young tableaux,

J. Combinatorial Theory, Ser. A, 17 (1974), 12-30.

5. P. Crawley and R. P. Dilworth, Algebraic Theory of Lattices, Prentice-Hall, Englewood Cliffs, NJ, 1973. 
6. G. Frobenius, Über die Charaktere der symmetrischen Gruppe, S.-B. Preuss. Akad. Wiss. (Berlin, 1900), 516-534.

7. E. R. Gansner, Matrix correspondences and the enumeration of plane partitions, Ph.D. Dissertation, M.I.T., February, 1978.

8. On the equality of two plane partition correspondences, Discrete Math., 30 (1980), 121-132.

9. - The Hillman-Grassl correspondence and the enumeration of reverse plane partitions, J. Combinatorial Theory Ser. A, to appear.

10. - The enumeration of plane partitions via the Burge correspondence, preprint.

11. C. Greene, An extension of Schensted's theorem, Advances in Math., 14 (1974), 254265 .

12. Some order-theoretic properties of the Robinson-Schensted correspondence, in "Combinatoire et Représentation du Groupe Symétrique", D. Foata, ed., Lecture Notes in Mathematics No. 579, Springer-Verlag, Berlin, 1977, 114-120.

13. A. P. Hillman and R. M. Grass1, Reverse plane partitions and tableaux hook numbers, J. Combinatorial Theory, Ser. A, 21 (1976), 216-221.

14. - Functionx on tableaus frames, Discrete Math., 25 (1979), 245-255.

15. D. E. Knuth, Permutations, matrices, and generalized Young tableaux, Pacific J. Math., 34 (1970), 709-727.

16. The Art of Computer Programming, Vol. 3, Addison-Wesley, Reading, Mass., 1973.

17. C. L. Liu, Topics in Combinatorial Mathematıcs, Math. Assoc. Amer., Washington, DC, 1972.

18. P. A. MacMahon, Combinatory Analysis, Vol. 2, Cambridge University Press, 1916; reprinted by Chelsea, New York, 1960.

19. G. de B. Robinson, On the representations of the symmetric group, Amer. J. Math., 60 (1938), 745-760.

20. C. Schensted, Longest increasing and decreasing subsequences, Canad. J. Math., 13 (1961), 179-191.

21. M.-P. Schützenberger, Quelques remarques sur une construction de Schensted, Math. Scand., 12 (1963), 117-128.

22. - Promotion des morphismes d'ensembles ordonées, Discrete Math., 2 (1972), 73-94.

23. R. P. Stanley, Theory and applications of plane partitions $I$, II, Studies in Applied Math., 50 (1971), 167-188, 259-279.

24. G. P. Thomas, On a construction of Schützenberger, Discrete Math., 17 (1977), 107-118.

25. On Schensted's construction and the multiplication of Schur functions, Advances in Math., 30 (1978), 8-32.

Received August 8, 1979 and in revised form June 26, 1980.

UNiversity of Illinois

Urbana, IL 61801

Current address: Bell Laboratories

Murry Hill, NJ 07974 



\section{PACIFIC JOURNAL OF MATHEMATICS}

\section{EDITORS}

DONALD BABBITT (Managing Editor)

University of California

Los Angeles, CA 90024

HUGo RossI

University of Utah

Salt Lake City, UT 84112

C. C. MOORE and ANDREW OGG

University of California

Berkeley, CA 94720
J. DUGUNDJI

Department of Mathematics

University of Southern California

Los Angeles, CA 90007

R. FinN and J. Milgram

Stanford University

Stanford, CA 94305

\section{ASSOCIATE EDITORS}
R. ARENS
E. F. BECKENBACH
B. H. NeumanN
F. WOLF
K. Yoshida

\section{SUPPORTING INSTITUTIONS}

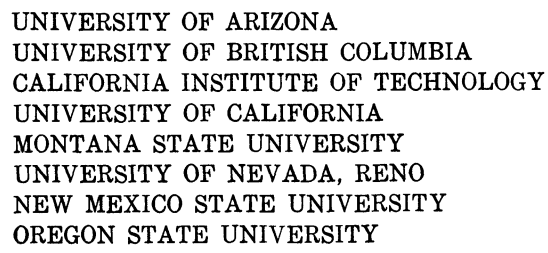

UNIVERSITY OF ARIZONA

UNIVERSITY OF BRITISH COLUMBIA CALIFORNIA INSTITUTE OF TECHNOLOGY

UNIVERSITY OF CALIFORNIA

MONTANA STATE UNIVERSITY

UNIVERSITY OF NEVADA, RENO

NEW MEXICO STATE UNIVERSITY

OREGON STATE UNIVERSITY

\author{
UNIVERSITY OF OREGON \\ UNIVERSITY OF SOUTHERN CALIFORNIA \\ STANFORD UNIVERSITY \\ UNIVERSITY OF HAWAII \\ UNIVERSITY OF TOKYO \\ UNIVERSITY OF UTAH \\ WASHINGTON STATE UNIVERSITY \\ UNIVERSITY OF WASHINGTON
}

The Supporting Institutions listed above contribute to the cost of publication of this Journal, but they are not owners or publishers and have no responsibility for its content or policies.

Mathematical papers intended for publication in the Pacific Journal of Mathematics should be in typed form or offset-reproduced, (not dittoed), double spaced with large margins. Please do not use built up fractions in the text of the manuscript. However, you may use them in the displayed equations. Underline Greek letters in red, German in green, and script in blue. The first paragraph or two must be capable of being used separately as a synopsis of the entire paper. Please propose a heading for the odd numbered pages of less than 35 characters. Manuscripts, in triplicate, may be sent to any one of the editors. Please classify according to the scheme of Math. Reviews, Index to Vol. 39. Supply name and address of author to whom proofs should be sent. All other communications should be addressed to the managing editor, or Elaine Barth, University of California, Los Angeles, California, 90024.

50 reprints to each author are provided free for each article, only if page charges have been substantially paid. Additional copies may be obtained at cost in multiples of 50 .

The Pacific Journal of Mathematics is issued monthly as of January 1966. Regular subscription rate: $\$ 102.00$ a year (6 Vols., 12 issues). Special rate: $\$ 51.00$ a year to individual members of supporting institutions.

Subscriptions, orders for numbers issued in the last three calendar years, and changes of address shoud be sent to Pacific Journal of Mathematics, P.O. Box 969, Carmel Valley, CA 93924, U.S.A Old back numbers obtainable from Kraus Periodicals Co., Route 100, Millwood, NY 10546.

\section{PUBLISHED BY PACIFIC JOURNAL OF MATHEMATICS, A NON-PROFIT CORPORATION}

Printed at Kokusai Bunken Insatsusha (International Academic Printing Co., Ltd.). 8-8, 3-chome, Takadanobaba, Shinjuku-ku, Tokyo 160, Japan. 


\section{Pacific Journal of Mathematics}

Vol. 92, No. $2 \quad$ February, 1981

Bruce Allem Anderson and Philip A. Leonard, Sequencings and Howell designs

Kevin T. Andrews, Representation of compact and weakly compact

operators on the space of Bochner integrable functions . . . . . . . . 257

James Glenn Brookshear, On the structure of hyper-real $z$-ultrafilters . . . . . 269

Frank John Forelli, Jr., A necessary condition on the extreme points of a class of holomorphic functions. II ...................... 277

Richard J. Friedlander, Basil Gordon and Peter Tannenbaum, Partitions of groups and complete mappings ......................... 283

Emden Robert Gansner, Matrix correspondences of plane partitions ......295

David Andrew Gay and William Yslas Vélez, The torsion group of a radical extension ..........................................

André (Piotrowsky) De Korvin and C. E. Roberts, Convergence theorems for some scalar valued integrals when the measure is Nemytskii ...... 329

Takaŝi Kusano and Manabu Naito, Oscillation criteria for general linear ordinary differential equations $\ldots \ldots \ldots \ldots \ldots \ldots \ldots \ldots \ldots \ldots \ldots \ldots \ldots \ldots \ldots \ldots$

Vo Thanh Liem, Homotopy dimension of some orbit spaces .......... 357

Mark Mahowald, $b o$-resolutions . . . . . . . . . . . . . . . . . . . 365

Jan van Mill and Marcel Lodewijk Johanna van de Vel, Subbases, convex

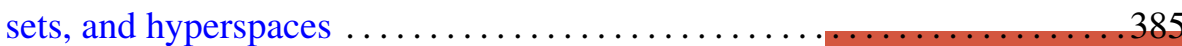

John F. Morrison, Approximations to real algebraic numbers by algebraic numbers of smaller degree $\ldots \ldots \ldots \ldots \ldots \ldots \ldots \ldots \ldots \ldots \ldots \ldots \ldots$

Caroline Series, An application of groupoid cohomology . . . . . . . . . . 415

Peter Frederick Stiller, Monodromy and invariants of elliptic surfaces . . . 433 Akihito Uchiyama, The factorization of $H^{p}$ on the space of homogeneous

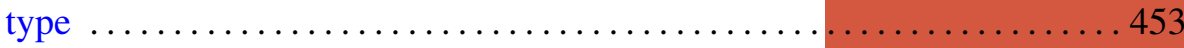

Warren James Wong, Maps on simple algebras preserving zero products.

II. Lie algebras of linear type 